\title{
Elastic Scattering in Kane Type Semiconductor Circular Dots
}

\author{
A.M. BABAnLI ${ }^{a, b}$, E. ARTUNÇ ${ }^{b}$ AND T.F. KASAlAK ${ }^{c, *}$ \\ ${ }^{a}$ Institute of Physics, Azerbaijan National Academy of Sciences, 370143 Baku, Azerbaijan \\ ${ }^{b}$ Department of Physics, University of Süleyman Demirel, 32260 Isparta, Turkey \\ ${ }^{c}$ Department of Informatics, University of Akdeniz, 07058 Antalya, Turkey \\ (Received June 4, 2014; revised version December 9, 2014; in final form January 27, 2015)
}

\begin{abstract}
In this paper we have investigated the scattering of electrons by a circular narrow penetrable $\delta$-type potential barrier in $\mathrm{A}^{3} \mathrm{~B}^{5}$ type semiconductors by using three-band Kane model. By using the Kane equations with the continuous conditions of the wave functions and flux discontinuous at the interface of two circular dots, we have analytically calculated the total cross-section and the Boltzmann conductivity for the semiconductor quantum rings with delta potential barrier. It has been shown that the quasi-bound states appear as peaks in the cross-section.
\end{abstract}

DOI: 10.12693 /APhysPolA.127.811

PACS: 73.22.Pr, 73.20.D-

\section{Introduction}

In recent years, there have been intensive studies in connection with features of quantum nanostructures such as quantum wells, wires and dots [1]. Electrons traversing through ultra-small semiconductor microstructures give rise to a fascinating regime of quantum transport [2]. Recent progress on the synthetic chemistry of semiconductor nanocrystals has made it possible to access high quality semiconductor nanocrystals with controlled size, shape, and optical properties. The low-dimensional semiconductor nanostructures, such as quantum dots, quantum wires, and quantum wells, have been applied in several areas. Current technologies can be used to fabricate quantum dots of various types, including both core-shell ones [3] and those embedded in a matrix made of a different semiconductor material. It has been shown experimentally that core-shell quantum dots can be effectively used in biology and medicine [4-6]. Core-shell quantum dots are primarily used for in vivo imaging and identifying living cells. Core-shell quantum dots have a nanometer sized semiconductor core coated with a thin layer of a outer shell material. The carrier spectrum in such a dot strongly depends on the core radius and the thickness of the coating shell. The scattering of two dimensional massless Dirac electrons was investigated [7] in presence of a random selected array of circular mass barriers.

In this paper, the electron scattering problem was investigated for a $\delta$-type potential barrier in narrowgap semiconductors circular dots using three-band Kane model, and calculated the cross-section and the Boltzmann conductivity to demonstrate how possible the quasi-bound states reveal themselves in the scattering cross-section and the Boltzmann conductivity.

The three-band Kane model faithfully describes the wave functions and spectrum of the carriers in narrowgap $\mathrm{A}^{3} \mathrm{~B}^{5}$ semiconductors. The energy spectrum is

\footnotetext{
* corresponding author; e-mail: tfatih@akdeniz.edu.tr
}

parabolic only in the $\mathrm{A}^{3} \mathrm{~B}^{5}$ type semiconductors near the bottom of their conduction band. In the majority of other substances, non-parabolicity of the energy spectrum plays an important role. While considering the non-parabolicity of the electron dispersion in narrow and medium gap semiconductors the coupling of conduction and valance bands should be taken into account. This is the purpose of our work.

\section{Theory}

The model which is considered in this study consists of an open circular quantum dot which is a narrow-gap semiconductor having radius $\rho$. It is surrounded by a very thin insulating circular layer and placed in the same semiconductor region. We treat the thin insulating circular layer as a $\delta$-type potential at $\rho=a$ (see Fig. 1), $U(\rho)=U a \Omega \delta(\rho-a)$, where $\delta(\rho-a)$ is the Dirac delta function. We study the scattering of the Kane electrons by extremely narrow penetrable circle (i.e., a $\delta$-type potential). Now it is assumed that an electron with energy $E$ travels from III. section of the circular dot through I. section of the circular dot.

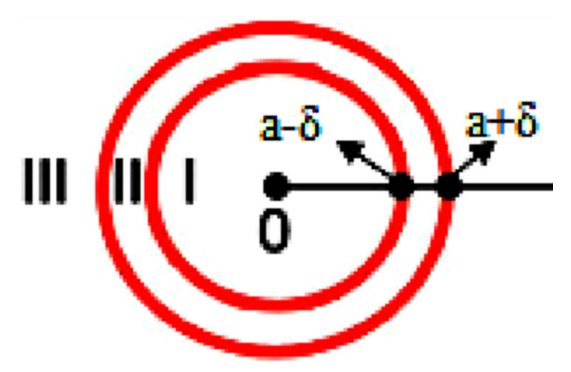

Fig. 1. Penetrable circular mass barrier model.

According to the standard theory of scattering wave functions are as follows:

$$
\Psi=\mathrm{e}^{\mathrm{i} k x}+\frac{f(\theta)}{\sqrt{\rho}} \mathrm{e}^{\mathrm{i} k \rho}
$$

where they consist on the incident wave in the $x$ direction 
and the scattered section. As the incoming wave-function part is normalized to unit flow density, the differential cross-section is equal to the radial flow of electrons corresponding to the scattered wave-function part, namely

$$
\sigma=\int_{0}^{2 \pi}|f(\theta)|^{2} \mathrm{~d} \theta
$$

The transport cross-section

$$
\sigma_{\text {tr }}=\int_{0}^{2 \pi}|f(\theta)|^{2}(1-\cos \theta) \mathrm{d} \theta .
$$

In the calculations in this paper we use the nonparabolic approximation for the energy dependences of the electronic effective mass, proposed in Refs. [8, 9].

In circular coordinates, it becomes

$$
\left(-\frac{\hbar^{2}}{2 m(E)} \Delta_{2}-E+U a \delta(\rho-a)\right) \Psi(\rho, \phi)=0,
$$

where

$$
\frac{\hbar^{2}}{2 m(E)}=\frac{P^{2}}{3}\left(\frac{2}{E+E_{\mathrm{g}}}+\frac{1}{E+E_{\mathrm{g}}+\Delta}\right) .
$$

$P$ is the matrix element which indicates the interaction between the conduction band and the valence band. The matrix element $P$ is expressed in terms of the effective mass at conduction band bottom $m_{n}$ and other parameters ( $E_{\mathrm{g}}$ is the band gap and $\Delta$ is the spin-orbit splitting energy of the valence bands)

$$
\frac{P^{2}}{3}=\frac{\hbar^{2}}{2 m_{n}} \frac{E_{\mathrm{g}}\left(E_{\mathrm{g}}+\Delta\right)}{\left(3 E_{\mathrm{g}}+2 \Delta\right)},
$$

where $\Delta_{2}$ is two-dimensional Laplacian. In polar coordinates we expand the total wave functions into partial waves

$$
\Psi(\rho, \varphi)=\sum_{l} \exp (\mathrm{i} l \varphi) R_{l}(\rho) .
$$

The radial function $R_{l}(\rho)$ can be used to meet differential Eq. (8):

$$
\begin{aligned}
& {\left[\frac{\hbar^{2}}{2 m(E)}\left(\frac{d^{2}}{d \rho^{2}}+\frac{1}{\rho} \frac{d}{d \rho}-\frac{l^{2}}{\rho^{2}}\right)+E-U a \delta(\rho-a)\right]} \\
& \quad \times R_{l}(\rho)=0 .
\end{aligned}
$$

The regular solution at the origin would be as follows:

$$
R_{l}(\rho)=T J_{l}(k \rho) .
$$

The solution for the outer section is a linear combination of the cylindrical Bessel functions that can be written as

$$
R_{l}(\rho)=A_{l}\left[J_{l}(k \rho) \cos \delta_{l}+Y_{l}(k \rho) \sin \delta_{l}\right],
$$

where $J_{l}(k \rho)$ and $Y_{l}(k \rho)$ are Bessel functions of first and second kind, $\delta_{l}$ is phase shift and using the differential Eq. (8), we found the $k$ wave vector as Eq. (11):

$$
k=\sqrt{\frac{3}{P^{2}} \frac{E\left(E+E_{\mathrm{g}}\right)\left(E+E_{\mathrm{g}}+\Delta\right)}{\left(3 E+3 E_{\mathrm{g}}+2 \Delta\right)}} .
$$

The radial wave function must be continuous at the potential singularity but first derivative of the function must be discontinuous at $\rho=a$. Thus, we have

$$
R_{a+}(\rho)=R_{a-}(\rho),
$$

$$
\frac{d}{d \rho} R_{a+}-\frac{d}{d \rho} R_{a-}=\frac{2 m(E)}{\hbar^{2}} \operatorname{UaR}(a) .
$$

To determine the phase shift, we should take into consideration the boundary conditions (12), (13) and the Wronskian Eqs. (14):

$$
Y_{l}^{\prime}(z) J_{l}(z)-J_{l}^{\prime}(z) Y_{l}(z)=\frac{2}{\pi z} .
$$

We get

$$
\tan \delta_{l}=\frac{J_{l}(k \rho)}{\frac{2}{\Omega \pi \frac{3 E_{\mathrm{g}}+2 \Delta}{E_{\mathrm{g}}\left(E_{\mathrm{g}}+\Delta\right)} \frac{\left(E+E_{\mathrm{g}}\right)(E+E \mathrm{~g}+\Delta)}{3 E+3 E_{\mathrm{g}}+2 \Delta} J_{l}(k \rho)}-Y_{l}(k \rho)},
$$

where $\Omega=\frac{2 m_{n} U a^{2}}{\hbar^{2}}$ characterizes the penetrability of the barrier. In case of $\Delta \rightarrow \infty$ formula (15) gives the same results with equation A6 in [7]. The value $\Omega \rightarrow \infty$ corresponds to a completely impenetrable barrier, while the value $\Omega=0$ corresponds to the case of complete penetration, or the absence of any scatter.

Usually the exclusion of the incoming plane wave from the total wave function (7) is done in the asymptotic region where $k \rho \rightarrow \infty$. Here we use the asymptotic of the Bessel functions

$$
\begin{aligned}
& J_{l}(k \rho) \approx \sqrt{\frac{2}{\pi k \rho}} \cos \left(k \rho-\frac{\pi}{2} l-\frac{\pi}{4}\right), \\
& Y_{l}(k \rho) \approx \sqrt{\frac{2}{\pi k \rho}} \sin \left(k \rho-\frac{\pi}{2} l-\frac{\pi}{4}\right)
\end{aligned}
$$

that components of the total wave functions allow us to write as

$$
\begin{aligned}
& \sum_{l} \exp (\mathrm{i} l \varphi) R_{l}(\rho)= \\
& \quad \sqrt{\frac{2}{\pi k \rho}} \sum_{l} \exp (\mathrm{i} l \varphi) A_{l} \cos \left(k \rho-\frac{\pi}{2} l-\frac{\pi}{4}-\delta_{l}\right)
\end{aligned}
$$

In this incoming plane wave can be presented as in the asymptotic region

$$
\begin{aligned}
& \mathrm{e}^{\mathrm{i} k x}=\sum_{l=-\infty}^{\infty} \mathrm{i}^{l} \mathrm{e}^{\mathrm{i} l \theta} J_{l}(k \rho) \approx \\
& \quad \sqrt{\frac{2}{\pi k \rho}} \sum_{l=-\infty}^{\infty} \mathrm{i}^{l} \mathrm{e}^{\mathrm{i} l \theta} \cos \left(k \rho-\frac{\pi}{2} l-\frac{\pi}{4}\right)
\end{aligned}
$$

by replacing these equations in (1), we get Eq. (19):

$$
\begin{gathered}
\sqrt{\frac{2}{\pi k \rho}} \sum_{l=-\infty}^{\infty} \mathrm{i}^{l} \mathrm{e}^{\mathrm{i} l \theta} \cos \left(k \rho-\frac{\pi}{2} l-\frac{\pi}{4}\right)+\frac{f(\theta)}{\sqrt{\rho}} \mathrm{e}^{\mathrm{i} k \rho}= \\
\sqrt{\frac{2}{\pi k \rho}} \sum_{l=-\infty}^{\infty} \mathrm{e}^{\mathrm{i} l \theta} A_{l} \cos \left(k \rho-\frac{\pi}{2} l-\frac{\pi}{4}-\delta_{l}\right),
\end{gathered}
$$

which may be written as

$$
\begin{aligned}
& \sqrt{\frac{2}{\pi k \rho}} \sum_{l=-\infty}^{\infty} \mathrm{i}^{l} \mathrm{e}^{\mathrm{i} l \theta} \frac{1}{2}\left(\mathrm{e}^{\mathrm{i}\left(k \rho-\frac{\pi}{2} l-\frac{\pi}{4}\right)}+\mathrm{e}^{-\mathrm{i}\left(k \rho-\frac{\pi}{2} l-\frac{\pi}{4}\right)}\right) \\
& +\frac{f(\theta)}{\sqrt{\rho}} \mathrm{e}^{\mathrm{i} k \rho}=\sqrt{\frac{2}{\pi k \rho}} \sum_{l=-\infty}^{\infty} \mathrm{e}^{\mathrm{i} l \theta} A_{l} \\
& \times \frac{1}{2}\left(\mathrm{e}^{\mathrm{i}\left(k \rho-\frac{\pi}{2} l-\frac{\pi}{4}-\delta_{l}\right)}+\mathrm{e}^{-\mathrm{i}\left(k \rho-\frac{\pi}{2} l-\frac{\pi}{4}-\delta_{l}\right)}\right) .
\end{aligned}
$$


The coefficients of $\mathrm{e}^{\mathrm{i} k \rho}$ and $\mathrm{e}^{-\mathrm{i} k \rho}$ in Eq. (19) must be equal. From it we find expression for

$$
\begin{aligned}
& A_{l}=\mathrm{i}^{l} \mathrm{e}^{-\mathrm{i} \delta_{l}}, \\
& f(\theta)=\frac{1}{\sqrt{2 \pi \mathrm{i} k}} \sum_{l=-\infty}^{\infty} \mathrm{e}^{\mathrm{i} l \theta}\left[\exp \left(2 i \delta_{l}-1\right)\right] .
\end{aligned}
$$

Inserting the expression into Eq. (2) we obtain the total cross-section as

$$
\sigma=\int_{0}^{2 \pi}|f(\theta)|^{2} \mathrm{~d} \theta=\frac{4}{k} \sum_{l} \sin ^{2} \delta_{l} .
$$

The transport scattering cross-sections can be expressed in terms of $\delta_{l}$ as follows from Ref. [10]:

$$
\sigma_{\mathrm{tr}}=\frac{2}{k} \sum_{l} \sin ^{2}\left(\delta_{l+1}-\delta_{l}\right) \text {. }
$$

The presented (24) in the formula can be used in order to calculate the Boltzmann conductivity

$$
\sigma_{\mathrm{B}}=\frac{n e^{2}}{m} \tau_{\mathrm{tr}}
$$

where the transport relaxion time equals

$$
\frac{1}{\tau_{\mathrm{tr}}}=N_{\mathrm{i}} v \sigma_{\mathrm{tr}},
$$

where $N_{\mathrm{i}}$ is the areal impurity density, $v$ is the electron velocity. The Boltzmann conductivity can written as after using (26):

$$
\sigma_{\mathrm{B}}=\frac{n e^{2}}{N_{\mathrm{i}} m v \sigma_{\mathrm{tr}}}=\sigma_{0} \frac{\pi n}{N_{\mathrm{i}} \sum_{l} \sin ^{2}\left(\delta_{l+1}-\delta_{l}\right)},
$$

where $\sigma_{0}=\frac{e^{2}}{2 \pi \hbar}, n$ is the number of conduction electrons in unit area and $\delta_{l}$ is the phase shift of $i$-th partial wave.

\section{Results and discussion}

We present scattering of the Kane electrons by a penetrable circle for model consisting of InSb/very thin insulating circular layer / InSb system. We used the value of the effective mass of electrons $m=0.016 m_{0}$ and band gaps $\varepsilon_{\mathrm{g}}$ for $\operatorname{InSb} \varepsilon_{\mathrm{g}}=0.23 \mathrm{eV}$, where $m_{0}$ is the mass of free electrons provided in Ref. [9]. The inner section radius of circular dots was taken equal to $\rho=200 \AA$.

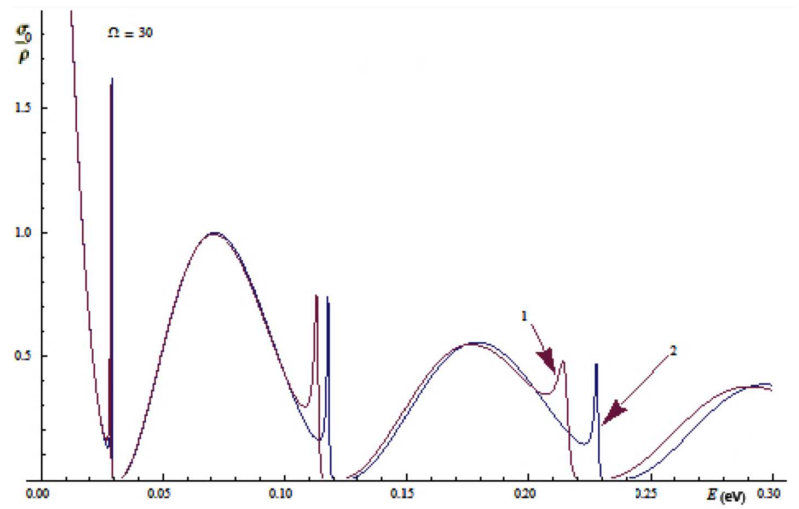

Fig. 2. The $l=0$ contribution to the total crosssection for Kane (curve 1) and Schrödinger (curve 2) electrons scattered on penetrable circular potentials.

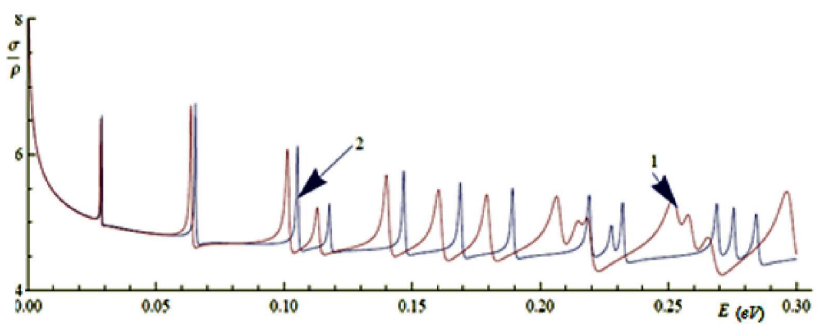

Fig. 3. The dependence of total cross-section as a function of the electron energy for Kane (curve 1) and Schrodinger (curve 2) electrons scattered on penetrable circular potentials.

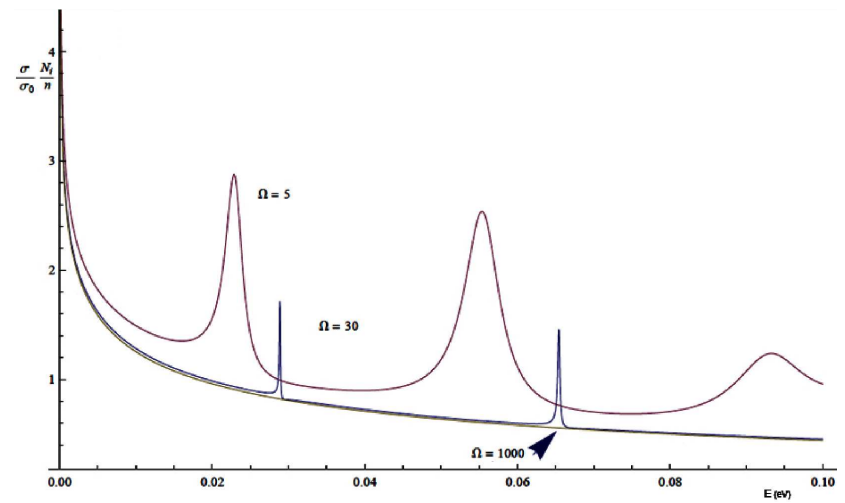

Fig. 4. The Boltzmann conductivity as a functions of the Electron energy for different values $\Omega$.

The $l=0$ contribution of the total cross-section for Kane (curve 1) and Schrödinger (curve 2) electrons scattered on penetrable circular delta potentials for $\Omega=30$ is shown by Fig. 2. The term Kane type electrons can be defined as semiconductors which are applied to Kane model and term Schrödinger electrons defined as semiconductors which are applied to simple parabolic model.

Therefore electrons scattering of Kane is shown by the curve 1 , but the curve 2 shows the parabolic band model. Narrow peaks appear close to the positions of the bound states of a dot that are defined by the equation $J_{l}(k \rho)=$ 0 . In the case of Kane's dispersions the energy of quasibound states are shifted to the left.

In Fig. 3 we plot the total cross-section as a function of $E$. The resonances are shown up as small peaks, corresponding to the quasi-bound states. In Fig. 4, we plot the Boltzmann conductivity as a functions of the electron energy for different values of $\Omega$. We observe many resonances, which diminish for increasing electron energy $E$. These resonances correspond to quasi-bound states. The value $\Omega \rightarrow \infty$ corresponds to a completely impenetrable circle and the absence of any scattered. (Figure 4 also shows that in this case value $\Omega=1000$.)

\section{Conclusion}

In this work we studied elastic scattering with cylindrical $\delta$-type potential barrier in narrow-gap semiconductors ring. For this purpose, we investigated the scattering of 
Kane electrons by circular penetrable scatterer. The total cross-section for scattering on penetrable delta barriers exhibits resonances due to the presence of quasibound states in the barriers that show up as peaks in the cross-sections. The obtained results were compared to results for scattering of Schrödinger electrons by similar scatterers. This was demonstrated by comparing figures. There is an essential difference in the energy dependence of the cross-section between Kane and Schrödinger electrons. Finally we calculated the Boltzmann conductivity and show that the Boltzmann conductivity as function of the electron energy exhibits a pronounced oscillatory structure due to the presence of quasi-bound states.

\section{References}

[1] T. Chakraborty, Quantum Dots, Elsevier, Amsterdam 1999.

[2] C.W.J. Beenakker, H. van Houten, Solid State Phys. 44, 1 (1991).
[3] M.A. Stroscio, M. Dutta, Biological Nanostructures and Applications of Nanostructures in Biology: Electrical, Mechanical, and Optical Properties, Kluwer, New York 2004

[4] W.C.W. Chan, S. Nie, Science 281, 2016 (1998).

[5] X. Michalet, F.F. Pinaud, L.A. Bentolila, J.M. Tsay, S. Doose, J.J. Li, G. Sundaresan, A.M. Wu, S.S. Gambhir, S. Weiss, Science 307, 538 (2005).

[6] X. Gao, Y. Cui, R.M. Levenson, L.W.K. Chung, S. Nie, Nat. Biotechnol. 22, 969 (2004).

[7] M.R. Masir, A. Matulis, F.M. Peeters, Phys. Rev. B 84, 245413 (2011).

[8] E.L. Ivchenko, Optical Spectroscopy of Semiconductor Nanostructures, Alpha Science Int., Harrow 2005.

[9] B.M. Askerov, Kinetic Effects in Semiconductors, World Sci., Nauka, Leningrad 1985.

[10] D.S. Novikov, Phys. Rev. B 76, 245435 (2007). 